\title{
Coherent control of atomic excitation using off-resonant strong few-cycle pulses
}

\author{
Pankaj K. Jha, ${ }^{1, *}$ Hichem Eleuch, ${ }^{1,2, \dagger}$ and Yuri V. Rostovtsev ${ }^{1,3}$ \\ ${ }^{1}$ Institute for Quantum Science and Engineering and Department of Physics and Astronomy, \\ Texas A\&M University, College Station, Texas 77843, USA \\ ${ }^{2}$ Institut National des Sciences Appliquées et de Technologie, 1080 Tunis, Tunisia \\ ${ }^{3}$ Department of Physics, University of North Texas, Denton, Texas 76203, USA
}

(Received 15 August 2010; published 20 October 2010)

\begin{abstract}
We study the dynamics of a two-level system driven by an off-resonance few-cycle pulse which has a phase jump $\phi$ at $t=t_{0}$, in contrast to many-cycle pulses, under the nonrotating-wave approximation (NRWA). We give a closed form analytical solution for the evolution of the probability amplitude $\left|C_{a}(t)\right|$ for the upper level. Using the appropriate pulse parameters like the phase jump $\phi$, jump time $t_{0}$, pulse width $\tau$, frequency $\nu$, and Rabi frequency $\Omega_{0}$ the population transfer after the pulse is gone can be optimized and, for the pulse considered here, an enhancement factor of $10^{6}-10^{8}$ was obtained.
\end{abstract}

DOI: 10.1103/PhysRevA.82.045805

PACS number(s): 42.65.Re, 32.80.Qk, 42.50.-p, 03.65.-w

Modern pulsed lasers produce bursts of light that are both ultrashort and ultrastrong, exhibiting durations comparable to those of molecular vibrations and electric fields rivaling those near an atomic nucleus [1]. Attosecond lasers, emitting pulses with only a few optical cycles per pulse [2], hold the promise of controlling the phase difference between the carrier wave and its envelope [3].

Of current interest is the interaction between strong broadband electromagnetic fields and atoms, especially laser radiation that is tuned far from resonance. Short pulses can excite coherence on high-frequency transitions that may be used for efficient generation of extreme ultraviolet (XUV) radiation [4-6]. Shaped pulses can enhance transient population of excited states [7] or create optimal coherence in two-level systems (TLSs) [8]. Recently, we have found an analytical solution describing the dynamics of a two-level atom under the action of laser radiation with an arbitrary pulse shape and polarization [9]. Furthermore, we have studied two mechanisms of atomic excitation: multiphoton excitation, and breaking of adiabaticity [4], and we have shown [10] that the latter can be more efficient.

The interaction of such ultrashort pulses with a two-level atom under the rotating-wave approximation does not give us the complete picture since the variation of the atomic polarization and population within the optical cycle is not slow. Thus, we should not neglect the contribution of the counter-rotating terms in the Hamiltonian while studying few-cycle-pulse interactions with atomic systems [11-18]. On the other hand, if the fields are not too strong and the variation of the atomic polarization and population within the optical cycle is slow, the rotating-wave approximation (RWA) appears to be a good approximation.

In this brief report, we study the interaction of few-cycle pulses (in contrast to many-cycle pulses [19-21]) with a TLS. These pulses have a phase jump $\phi$ at $t=t_{0}$. Thus, they can be characterized by the parameters peak Rabi frequency $\Omega_{0}$, pulse

*pkjha@physics.tamu.edu

${ }^{\dagger}$ Current Address: Physics and Astronomy Department, College of Science, P. O. Box 2455, King Saud University, Riyadh 11451, Saudi Arabia. width $\tau$, carrier frequency $v$, phase jump $\phi$, and jump moment $t_{0}$ along with the pulse envelope (which we have considered Gaussian for the numerical simulation, see Fig. 1). We present an analytical solution for this problem. Using the appropriate characterizing parameters, the population transfer can be optimized and, for the pulse considered here, enhancement by a factor of $10^{6}-10^{8}$ was obtained [see Fig. 5(b)].

The equation of motion for the probability amplitudes for the states $|a\rangle$ and $|b\rangle$ of a two-level atom (TLA) interacting with a classical field is given as [22]

$$
\begin{gathered}
\dot{C}_{a}=i \frac{\wp \mathcal{E}(t)}{\hbar} \cos (v t) e^{i \omega t} C_{b}, \\
\dot{C}_{b}=i \frac{\wp^{*} \mathcal{E}(t)}{\hbar} \cos (v t) e^{-i \omega t} C_{a},
\end{gathered}
$$

where $\hbar \omega$ is the energy difference between two levels, $\wp$ is the atomic dipole moment, $E(t)=\mathcal{E}(t) \cos (v t)$. In the RWA we let $\cos (v t) e^{ \pm i \omega t} \rightarrow e^{ \pm i \Delta t} / 2$, where $\Delta=\omega-v$ [23] is the detuning from resonance. Introducing $\Omega(t)=\wp \mathcal{E}(t) / \hbar$, Eqs. (1) reduces to

$$
\begin{gathered}
\dot{C}_{a}=i \frac{\Omega(t)}{2} e^{i \Delta t} C_{b}, \\
\dot{C}_{b}=i \frac{\Omega^{*}(t)}{2} e^{-i \Delta t} C_{a},
\end{gathered}
$$

which have an integral of motion $\left|C_{a}\right|^{2}+\left|C_{b}\right|^{2}=1$. If we define a function $f(t)=C_{a}(t) / C_{b}(t)$, then Eqs. (2) yields the following Riccati Equation:

$$
\dot{f}+i \frac{\Omega^{*}(t)}{2} e^{-i \Delta t} f^{2}-i \frac{\Omega(t)}{2} e^{i \Delta t}=0 .
$$

Then, $\left|C_{a}(t)\right|=|f(t)| / \sqrt{1+|f(t)|^{2}}$. Alternatively, we can get a second-order linear differential equation for $C_{a}(t)$ from Eqs. (2):

$$
\ddot{C}_{a}(t)-\left[i \Delta+\frac{\dot{\Omega}(t)}{\Omega(t)}\right] \dot{C}_{a}(t)+\frac{|\Omega(t)|^{2}}{4} C_{a}(t)=0 .
$$

In this article, we will work without the RWA, hence the Riccati Eq. (3) takes the form

$$
\dot{f}+i \Omega^{*}(t) \cos (v t) e^{-i \omega t} f^{2}-i \Omega(t) \cos (v t) e^{i \omega t}=0 .
$$




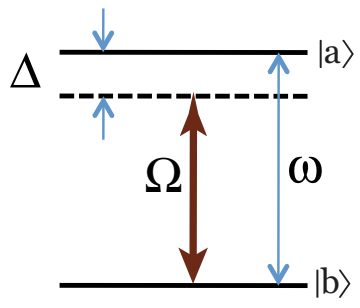

(a)

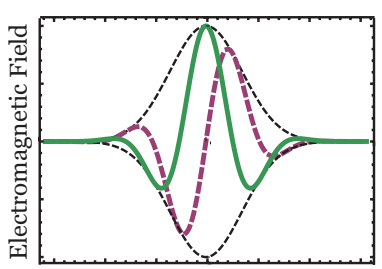

(b)
FIG. 1. (Color online) (a) Two-level atomic system, atomic transition frequency $\omega=\omega_{a}-\omega_{b}$, detuning $\Delta=\omega-v$, and Rabi frequency $\Omega(t)=\wp \mathcal{E}(t) / \hbar$. (b) Few-cycle sine (dashed line) and cosine (solid line) pulse with Gaussian envelope.

The approximate solution for Eq. (5), in terms of the tip angle $\theta$, is given as [9]

$$
\begin{aligned}
f(t)= & i \int_{-\infty}^{t} d t^{\prime}\left\{\left[\frac{d \theta\left(t^{\prime}\right)}{d t^{\prime}}-\theta^{2}\left(t^{\prime}\right) \frac{d \theta^{*}\left(t^{\prime}\right)}{d t^{\prime}}\right]\right. \\
& \left.\times \exp \left[2 \int_{t^{\prime}}^{t} \theta\left(t^{\prime \prime}\right) \dot{\theta}^{*}\left(t^{\prime \prime}\right) d t^{\prime \prime}\right]\right\},
\end{aligned}
$$

where the tip angle $\theta(t)$ has been defined as

$$
\theta(t)=\int_{-\infty}^{t} \Omega\left(t^{\prime}\right) \cos \left(v t^{\prime}\right) e^{i \omega t^{\prime}} d t^{\prime} .
$$

From Eq. (7) we can obtain $\left|C_{a}(t)\right|=|f(t)| / \sqrt{1+|f(t)|^{2}}$. To see how well the approximate solution works, we have plotted the probability amplitude $\left|C_{a}(\infty)\right|$ for a complex pulse shape given by $\Omega(t)=\Omega_{0}[\operatorname{sech}(\alpha t)+\operatorname{sech}(\alpha t-3)]$ (see Fig. 2 ). The numerical simulation (dashed line) and analytical solution (solid line) shown in Figs. 2(a) and 2(b) are nearly identical.

\section{A. Pulse with phase jump}

In this section we investigate the dynamics of a two-level atom subjected to a few-cycle pulse with a phase jump at an arbitrary time $t=t_{0}$. Let us define the Rabi frequency $\Omega(t)$ for our model as

$$
\Omega(t)= \begin{cases}\Omega_{-}(t) & \text { if } t<t_{0} \\ \Omega_{+}(t) & \text { if } t \geqslant t_{0}\end{cases}
$$

where $\Omega_{+}(t)=e^{i \phi} \Omega_{-}(t)$ and $\phi$ is the phase jump introduced into the electromagnetic field at $t=t_{0}$. Equivalently, the tip angle define by Eq. (7) takes the form

$$
\theta(t)= \begin{cases}\theta_{-}(t) & \text { if } t<t_{0} \\ \theta_{+}(t) & \text { if } t \geqslant t_{0}\end{cases}
$$

From the definition of the Rabi frequency [Eq. (8)], we can easily see that $\theta_{+}=e^{i \phi} \theta_{-}$. The time evolution of our system is divided into two regimes $\left(-\infty, t_{0}\right)$ and $\left(t_{0}, \infty\right)$. In both these regimes, the functional form of the solutions remains the same. We can write

$$
f_{\phi}(t)= \begin{cases}f_{-}(t) & \text { if } t<t_{0} \\ f_{+}(t) & \text { if } t \geqslant t_{0}\end{cases}
$$
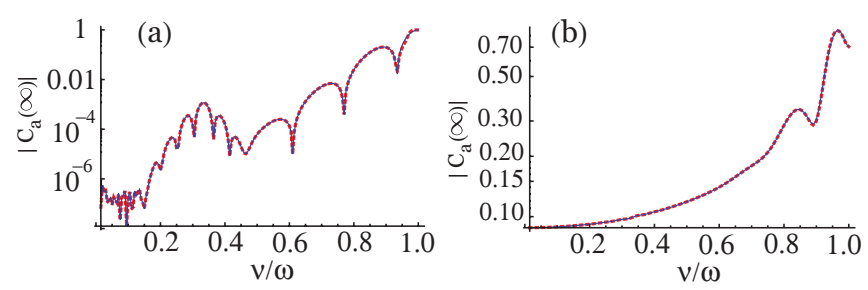

FIG. 2. (Color online) Population left in the upper level $|a\rangle$ after applying an $\Omega(t)=\Omega_{0}[\operatorname{sech}(\alpha t)+\operatorname{sech}(\alpha t-3)]$ pulse as a function of the frequency $v / \omega$ obtained by numerical solution of Eqs. (1) (dots) and using our approximate analytical result Eq. (6) (solid line). In calculations, we take $\Omega_{0}=0.04 \omega$ and $\alpha=0.075 \omega$. In (a) $\phi=0$, whereas in (b) $\phi=\pi$ and $t_{0}=0$.

Equation (6) is the solution for $\phi=0$ for the initial condition $f(-\infty)=0$. Using the same initial condition, we can safely write

$$
f_{-}(t)=i \int_{-\infty}^{t} d t^{\prime} \Phi_{-}\left(t^{\prime}\right) \exp \left[2 \int_{t^{\prime}}^{t} \zeta_{-}\left(t^{\prime \prime}\right) d t^{\prime \prime}\right],
$$

where

$$
\begin{gathered}
\Phi_{-}\left(t^{\prime}\right)=\left[\frac{d \theta_{-}\left(t^{\prime}\right)}{d t^{\prime}}-\theta_{-}^{2}\left(t^{\prime}\right) \frac{d \theta_{-}^{*}\left(t^{\prime}\right)}{d t^{\prime}}\right], \\
\zeta_{-}\left(t^{\prime \prime}\right)=\theta_{-}\left(t^{\prime \prime}\right) \dot{\theta}_{-}^{*}\left(t^{\prime \prime}\right) .
\end{gathered}
$$

As the functional form of $f_{+}(t)$ and $f_{-}(t)$ are the same, we can write

$$
f_{+}(t)=i \int_{t_{0}}^{t} d t^{\prime} \Phi_{+}\left(t^{\prime}\right) \exp \left[2 \int_{t^{\prime}}^{t} \zeta_{+}\left(t^{\prime \prime}\right) d t^{\prime \prime}\right]+c
$$

where $\Phi_{+}\left(t^{\prime}\right)=e^{i \phi} \Phi_{-}\left(t^{\prime}\right)$ and $\zeta_{+}\left(t^{\prime}\right)=\zeta_{-}\left(t^{\prime}\right)$. The constant $c$ can be obtained by demanding the continuity of $f_{\phi}(t)$ at $t=t_{0}$, which gives

$$
c=i \int_{-\infty}^{t_{0}} d t^{\prime} \Phi_{-}\left(t^{\prime}\right) \exp \left[2 \int_{t^{\prime}}^{t_{0}} \zeta_{-}\left(t^{\prime \prime}\right) d t^{\prime \prime}\right] .
$$

Population transferred to level $|a\rangle$ during the interaction is given as $\left|C_{a}(\infty)\right|^{2}=\left|f_{\phi}(\infty)\right|^{2} /\left[1+\left|f_{\phi}(\infty)\right|^{2}\right]$. In order to study the effect of the phase jump $\phi$, let us define a relative change in the amplitude

$$
r_{\phi}(t)=\left|\frac{f_{\phi}(t)-f(t)}{f(t)}\right| .
$$

Using Eqs. (11), (13), and (6) we get

$$
r_{\phi}(t)=\left|\frac{\left(e^{i \phi}-1\right) \int_{t_{0}}^{t} d t^{\prime} \Phi_{-}\left(t^{\prime}\right) \exp \left[2 \int_{t^{\prime}}^{t} \zeta_{-}\left(t^{\prime \prime}\right) d t^{\prime \prime}\right]}{\int_{-\infty}^{t} d t^{\prime} \Phi_{-}\left(t^{\prime}\right) \exp \left[2 \int_{t^{\prime}}^{t} \zeta_{-}\left(t^{\prime \prime}\right) d t^{\prime \prime}\right]}\right| .
$$

The asymptotic value $r_{\phi}(\infty)$ can be obtained by $t \rightarrow \infty$ in Eq. (16). We can easily see from Eq. (16) that $r_{\phi}(\infty)$ attains its maximum value for $\phi=\pi$.

\section{B. Effect of pulse parameters: numerical analysis}

In this section, we discuss the effect of pulse parameters such as the phase jump time $t_{0}$, pulse width $\tau$, detuning $\Delta$, and peak Rabi frequency $\Omega_{0}$ on the degree of excitation of the upper level $|a\rangle$. For computational purposes, we have considered 

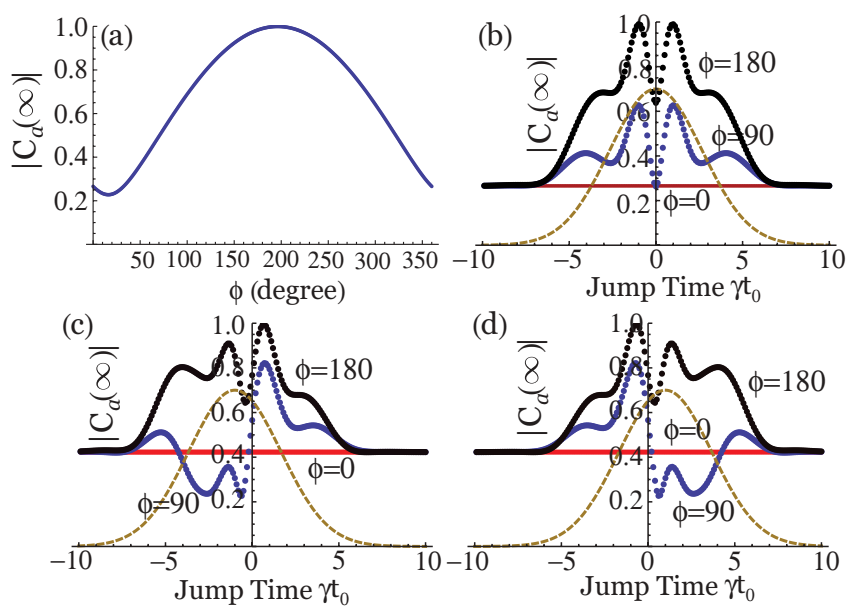

FIG. 3. (Color online) Effect of jump time $t_{0}$. (a) Probability amplitude $\left|C_{a}(\infty)\right|$ as a function of the phase jump $\phi$. The phase jump is introduced at the peak of the Gaussian envelope. (b) The symmetric influence on the degree of excitation with respect to the position of $t_{0}$. The symmetric response is lost for shifted Gaussian input pulses (c) and (d). For numerical calculations, we chose $\Omega_{0}=0.875 \omega$, $v=0.75 \omega, \alpha=0.331 \omega$, and $\gamma=1.25 \omega$.

a Gaussian pulse of the form $\Omega(t)=\Omega_{0} e^{-\alpha^{2} t^{2}}$ where $\alpha=$ $2 \sqrt{\ln 2} / \tau$ [ $\tau$ is the full width at half maximum (FWHM) of the pulse].

The main result showing the effect of relative position of $t_{0}$, with respect to the peak of the pulse, on the atomic excitation is shown in Figs. 3 and 7(a) where we have shown the dynamics of the two-level atom interacting with a few-cycle pulse with a phase jump. In Fig. 3(a), we have one such scenario with $\phi=\pi / 2$. Here, the phase jump is introduced in the field at the peak of the Gaussian envelope (i.e., $t_{0}=0$ ) and the probability amplitude $\left|C_{a}(\infty)\right|$ is plotted against the phase jump $\phi$. Interestingly, the difference in the maximum and

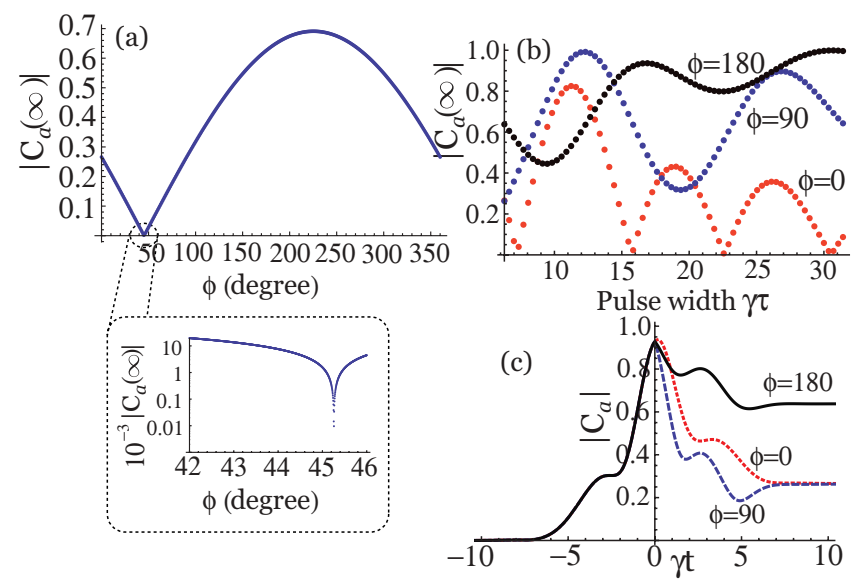

FIG. 4. (Color online) Effect of $\alpha$. (a) Probability amplitude $\left|C_{a}(\infty)\right|$ varies in the range from $10^{-5} \sim 0.7$. (b) Plot of $\left|C_{a}(\infty)\right|$ against normalized pulse width $\gamma \tau$ for fixed $\omega, \nu$, and $\Omega_{0}$, and three combinations of the phase jump: $\phi=0, \pi / 2$, and $\pi$. (c) The temporal evolution for the three combinations used in (b). For numerical simulation we chose $\Omega_{0}=0.875 \omega, v=0.75 \omega, \gamma=1.25 \omega$, and $\alpha=0.331 \omega$.
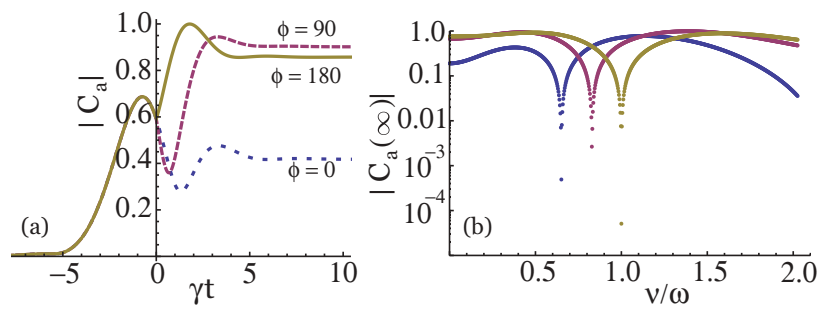

FIG. 5. (Color online) (a) Temporal behavior of $\left|C_{a}\right|$ for different combinations of $\phi$. (b) Plot of $\left|C_{a}(\infty)\right|$ against $\nu / \omega$. For numerical simulation we chose $\Omega_{0}=0.875 \omega, t_{0}=0, \gamma=1.25 \omega$, and $\alpha=$ $0.331 \omega$.

the minimum values corresponds to $\Delta \phi=\pi$. The symmetric nature of the atomic excitation is observed in Fig. 3(b) and in the contour plot in Fig. 7(a). With the shifted Gaussian pulse $\Omega=\Omega_{0} e^{-\alpha^{2}\left(t \pm t_{s}\right)^{2}}$ [see Figs. 3(c) and 3(d)], the symmetry is lost. Also the effect of the phase jump becomes significant for $t_{0}$ within the FWHM of the pulse and gradually decreases when $t_{0}$ is close to the tail of the pulse. An identical system response, for $\gamma t_{0} \approx 10$, is observed for the three combinations of the phase jump: $\phi=0, \pi / 2$, and $\pi$.

While investigating the effect of few-cycle pulses on atomic systems, the parameter $\alpha$ plays an important role for a given value of the carrier frequency $v$. It determines the number of cycles of the field in the pulse. The main results showing the effect of $\alpha$ or the pulse width $\tau$ is given in Fig. 4 and in the contour plot in Fig. 7(b). If we look at the inset of Fig. 4(a), we see that the probability amplitude $\left|C_{a}(\infty)\right|$ varies in the range from $10^{-5} \sim 0.7$. In Fig. 4(b), we have used three combinations of the phase jump $\phi(\phi=0, \pi / 2$, and $\pi)$ to study the effect of $\alpha$ on the degree of excitation. For a smaller pulse width $(2 \leqslant \gamma \tau \leqslant 15), \phi=\pi / 2$ creates more excitation than $\phi=0$ or $\pi$.

In order to study the effect of the detuning $\Delta$, we have plotted the response of the system in terms of $\left|C_{a}(\infty)\right|$ for the three combinations of $\phi$. Figure 5(a) shows the temporal behavior, while Fig. 5(b) gives information about the steadystate population. The probability amplitude $\left|C_{a}(\infty)\right|$ varies in the range from $4.4 \times 10^{-4} \sim 0.4$ for $\phi=0$ and $5 \times 10^{-5} \sim$ 0.9 for $\phi=\pi$. When $\left|C_{a}(\infty)\right|$ is $\sim 4.4 \times 10^{-4}$ for $\phi=0$, we have $\left|C_{a}(\infty)\right| \sim 1$ for $\phi=\pi$; thus we have an enhancement
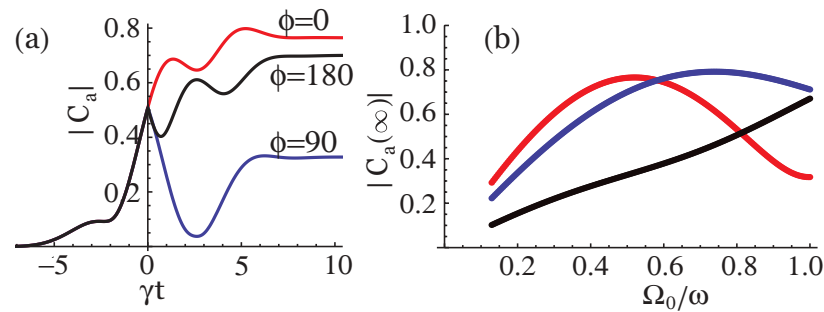

FIG. 6. (Color online) (a) Temporal behavior of $\left|C_{a}\right|$ for difference combination of $\phi$. (b) Plot of $\left|C_{a}(\infty)\right|$ against $\Omega_{0}$. For numerical simulation in (b), we chose a shifted Gaussian pulse with $t_{s}=$ $1, v=0.75 \omega, t_{0}=0, \gamma=1.25 \omega$, and $\alpha=0.331 \omega . \Omega_{0}=0.875 \omega$ for (a). 

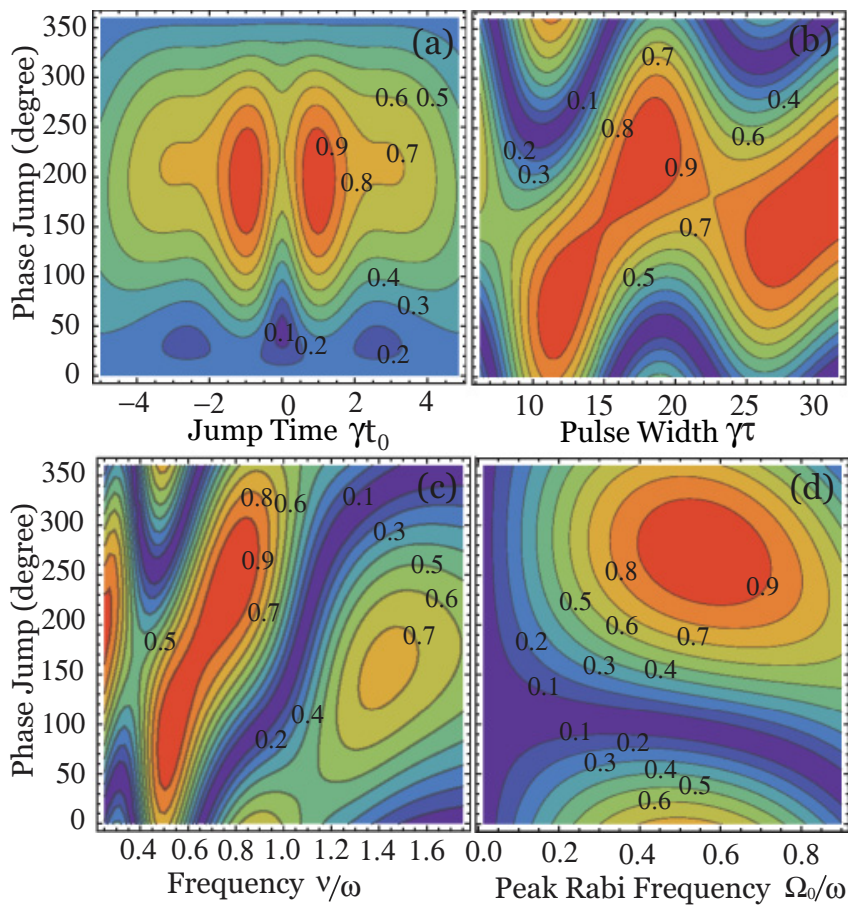

FIG. 7. (Color online) Contour plot showing the effect of pulse parameters such as $t_{0}, \tau, v$, and $\Omega_{0}$ on the population left in the excited states $|a\rangle$ in (a), (b), (c), and (d), respectively. The influence of the phase jump time $t_{0}$ is symmetric with respect to $t_{0}$, as shown in (a). The parameters used are $\Omega_{0}=0.875 \omega, v=0.75 \omega, \gamma=1.25 \omega, t_{0}=$ 0 , and $\alpha=0.331 \omega$ as required appropriately. For (c), we used $\alpha=$ $0.110 \omega$. by a factor of $10^{6}-10^{8}$ in the population transfer by introducing a phase jump of $\pi$ at the peak of the envelope function.

The effect of the peak Rabi frequency $\Omega_{0}$ on the degree of excitation of the upper level is shown in Fig. 6 and in the contour plot Fig. 7(d). Whereas Fig. 6(a) shows the temporal behavior of $\left|C_{a}\right|$, Fig. 6(b) gives information about the population left in the upper level after the pulse is gone. We see that, for some choices of $\Omega_{0}, \phi=0$ has the maximum effect whereas for other choices, $\phi=\pi / 2$ is dominant.

In conclusion, we have studied few-cycle pulses, with a phase jump $\phi$ at $t=t_{0}$, interacting with a two-level atom. This interaction is investigated without the rotating-wave approximation and we present an approximate solution for the probability amplitude $C_{a}(t)$ of the upper level. The approximate solution not only works well with multicycle pulses [9], but it is also in excellent agreement for few-cycles pulses (see Fig. 2). Using the appropriate pulse parameters $\phi, t_{0}, \alpha$, and $\Omega_{0}$ the population transfer, after the pulse is gone, can be optimized and, for the pulse considered here, an enhancement factor of $10^{6}-10^{8}$ was obtained [see Fig. 5(b)].

We thank M. O. Scully, L.V. Keldysh, and M. S. Zubairy for useful discussions and gratefully acknowledge support from NSF Grant No. EEC-0540832 (MIRTHE ERC), the Office of Naval Research (Grants No. N00014-09-1-0888 and No. N0001408-1-0948), the Robert A. Welch Foundation (Award No. A-1261), and partial support from the CRD. P.K.J. also acknowledges the Robert A. Welch Foundation and the HEEP Foundation for financial support.
[1] Martin Wegener, Extreme Nonlinear Optics: An Introduction (Berlin, Springer, 2005).

[2] T. Brabec and F. Krausz, Rev. Mod. Phys. 72, 545 (2000).

[3] A. Baltuska et al., Nature (London) 421, 611 (2003).

[4] M. O. Scully, Y. Rostovtsev, A. Svidzinsky, and Jun-Tao Chang, J. Mod. Opt. 55, 3219 (2008).

[5] P. K. Jha and Y. V. Rostovtsev, Phys. Rev. A 81, 033827 (2010).

[6] P. K. Jha and Y. V. Rostovtsev, Phys. Rev. A 82, 015801 (2010).

[7] N. Dudovich, D. Oron, and Y. Silberberg, Phys. Rev. Lett. 88, 123004 (2002).

[8] S. A. Malinovskaya, Opt. Commun. 282, 3527 (2009).

[9] Y. V. Rostovtsev, H. Eleuch, A. Svidzinsky, H. Li, V. Sautenkov, and M. O. Scully, Phys. Rev. A 79, 063833 (2009).

[10] Y. Rostovtsev, J. Mod. Opt. 55, 3149 (2008).

[11] L. W. Casperson, Phys. Rev. A 57, 609 (1998); 46, 401 (1992).

[12] S. Hughes, Phys. Rev. Lett. 81, 3363 (1998); Phys. Rev. A 62, 055401 (2000).

[13] N. Doslic, Phys. Rev. A 74, 013402 (2006).
[14] C. Jirauschek, L. Duan, O. D. Mücke, F. X. Krtner, M. Wegener, and U. Morgner, J. Opt. Soc. Am. B 22, 2065 (2005).

[15] R. Parzyński and M. Sobczak, J. Phys. B 37, 743 (2004).

[16] R. Parzyński and M. Sobczak, Opt. Commun. 228, 111 (2003).

[17] H. Li, V. A. Sautenkov, Y. V. Rostovtsev, M. M. Kash, P. M. Anisimov, G. R. Welch, and M. O. Scully, Phys. Rev. Lett. 104, 103001 (2010).

[18] P. K. Jha, Y. V. Rostovtsev, H. Li, V. A. Sautenkov, and M. O. Scully (unpublished).

[19] N. V. Vitanov, New J. Phys. 9, 58 (2007).

[20] B. T. Torosov and N. V. Vitanov, Phys. Rev. A 76, 053404 (2007).

[21] J. Qian, Y. Qian, M. Ke, Xun-Li Feng, C. H. Oh, and Y. Wang, Phys. Rev. A 80, 053413 (2009).

[22] M. O. Scully and M. S. Zubairy, Quantum Optics (Cambridge University Press, Cambridge, 1997).

[23] Here we use the convention that all frequencies are circular frequencies so that $\hbar v$ (not $h v$ ) is the photon energy. Some authors also use the definition of detuning $\Delta=v-\omega$. 